\title{
Interplay Between Spin-Orbit Coupling and Structural Deformations in Heavy Transition-Metal Oxides with Tetrahedral Coordination
}

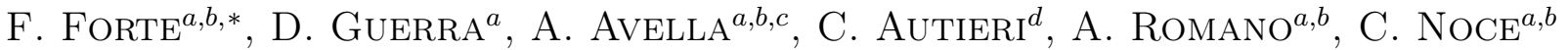 \\ ${ }^{a}$ Dipartimento di Fisica "E.R. Caianiello", Università degli Studi di Salerno, I-84084 Fisciano (SA), Italy \\ ${ }^{b}$ CNR-SPIN, UOS di Salerno, I-84084 Fisciano (SA), Italy \\ ${ }^{c}$ Unità CNISM di Salerno, Università degli Studi di Salerno, I-84084 Fisciano (SA), Italy \\ ${ }^{d}$ CNR-SPIN, UOS L'Aquila, Sede Temporanea di Chieti, 66100 Chieti, Italy
}

\begin{abstract}
We analyze the effects of a large spin-orbit coupling on the magnetic state of a $d^{1}$ transition-metal ion located in a tetrahedral environment. While in the ideal tetrahedral symmetry the spin-orbit coupling acts only as a perturbation on the atomic energy levels set by the crystal-field splitting, we demonstrate that its effects are strongly enhanced in the case of distorted geometries. In particular, we consider the specific case in which the tetrahedron is compressed along the $z$ direction, and show that, by increasing the degree of flattening, a large spin-orbit interaction (i) can induce a substantial anisotropic, unquenched orbital momentum and (ii) can affect the hierarchy of the lowest energy levels that are involved in the magnetic exchange.
\end{abstract}

DOI: 10.12693/APhysPolA.133.394

PACS/topics: 71.70.Ej, 75.25.Dk, 71.27.+a

\section{Introduction}

The collective ordering of magnetic moments in transition metal oxides (TMOs) and their mutual interactions have long been a central issue in condensed matter physics [1-6]. Most of this phenomenology is understood in terms of exchange interactions between spins. However, recent studies have revealed that $5 d$ TMOs may possess an unquenched orbital angular momentum, which is rigidly tied to the spin direction by the large intra-atomic spin-orbit coupling (SOC) expected for heavy transitionmetal atoms. In the case of a metal ion in an octahedral environment $\left(\mathrm{TMO}_{6}\right)$, mounting experimental evidence indicates that it is necessary to take into account a quite large SOC to achieve the correct magnetic state within the $t_{2 g}$ manifold. The most dramatic example occurs in $5 d^{5}$ iridates, where a quite strong SOC changes the character of the multiplet thus reducing the magnetic state of $\mathrm{Ir}^{4+}$ to an effective Kramers doublet with effective total angular momentum $j=1 / 2$ [7-12]. Conversely, the SOC in $5 d^{3}$ osmium-based compounds, albeit comparable in size to the one in $5 d^{5}$ iridates, can be treated perturbatively although it is anyhow necessary to account for the SOC-induced anisotropy, which is essential in the selection of the magnetic ground state [13-16].

Systems with active $e_{g}$ orbital degrees of freedom offer a completely different playground. It is known that the orbital momentum is fully quenched within the $e_{g}$ subspace, hence the SOC modifies the ground state only via virtual processes involving the $\mathrm{t}_{2 g}$ orbitals and, therefore, acting across the crystal field $(\mathrm{CF})$ splitting. However,

\footnotetext{
*corresponding author; e-mail: forte@sa.infn.it
}

this effect may be considerably enhanced in $5 d$ metal oxides containing $\mathrm{TMO}_{4}$ tetrahedra, since the CF splitting naturally favors the occupancy of the lower $e_{g}$ manifold, and the related CF energy $\left(\Delta_{t}\right)$, although being the largest energy scale, is relatively small in size (only $4 / 9$ of the corresponding octahedral one for the same ligands) $[17,18]$.

In order to understand the role that SOC plays on magnetism in such configurations, it is a good starting point to explore the interplay between electron-electron interactions, CF parameters and SOC at the TM ion site. In this paper, we analyze and discuss the specific case of an ionic $d^{1}$ configuration having spin $S=1 / 2$, where both the Coulomb interaction and the Hund's coupling within the $d$ subspace are neglected, and one is left with the simplified problem of competing $\mathrm{CF}, \mathrm{SOC}$ and structural distortions of the $\mathrm{TMO}_{4}$ tetrahedron. We demonstrate that structural deformations are a fundamental prerequisite to unveil the effects of SOC. We analyze the specific instance in which the tetrahedron is compressed along the $z$ direction and show that, due to the consequent symmetry breaking in the orbital space, one can get (i) an anisotropic, unquenched orbital momentum and (ii) an enhancement of the gap among the lowest energy levels involved in the magnetic exchange. The dependence upon the degree of flattening and the SOC parameters of the sizes of the gap is also reported and discussed.

\section{Spin-orbit coupling in the ideal tetrahedral symmetry}

The tetrahedral symmetry of the four O ligands surrounding the metal ion is shown in Fig. 1a. The energies of the $d$ orbitals are affected by the arrangement of the four negative charges leading to the CF splitting of the $d$ levels into two different subspaces. The top three are the 
$d_{x y}, d_{x z}$, and $d_{y z}$ orbitals, which span the $t_{2 g}$ sector and the bottom two are the $d_{x^{2}-y^{2}}$ and $d_{z^{2}}$ orbitals, which span the $e_{g}$ manifold (Fig. 1b).

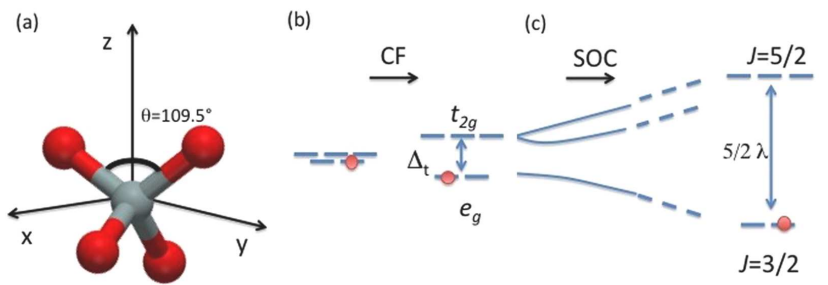

Fig. 1. (a) The TM ion is located at the origin of the coordinate system and the angle formed by the edges of the ideal tetrahedron is equal to $109.5^{\circ}$. (b) $e_{g}$ orbitals are shifted lower in energy with respect to $t_{2 g}$ orbitals by $\Delta_{t}$. (c) Hierarchy of the energy levels for an ideal tetrahedral structure in the presence of SOC.

Our simplified model Hamiltonian contains only the two interaction terms active at the TM ion site:

$$
H=H_{\mathrm{CF}}+H_{\mathrm{SOC}}
$$

with

$$
\begin{aligned}
& H_{\mathrm{CF}}=\sum_{\alpha} \epsilon_{\alpha} n_{\alpha}, \\
& H_{\mathrm{SOC}}=\lambda \boldsymbol{L} \cdot \boldsymbol{S},
\end{aligned}
$$

where $\alpha$ labels the five $d$ orbitals, $\epsilon_{y z}=\epsilon_{x z}=\epsilon_{x y} \equiv \epsilon_{t_{2 g}}$ and $\epsilon_{x^{2}-y^{2}}=\epsilon_{z^{2}} \equiv \epsilon_{e_{g}}$ stand for the on-site energies of the $t_{2 g}$ and the $e_{g}$ in the ideal tetrahedron, respectively, and $\lambda$ is the positive SOC parameter. Furthermore, we denote by $\boldsymbol{S}$ the spin one-half operator while $\boldsymbol{L}$ is the angular momentum operator for a single electron in one of the five-fold degenerate $d$-orbitals, whose components can be expressed as:

$$
\begin{aligned}
& L_{x}=\sqrt{3} \mathrm{i} c_{y z}^{\dagger} c_{z^{2}}+\mathrm{i}\left(c_{y z}^{\dagger} c_{x^{2}-y^{2}}-c_{x z}^{\dagger} c_{x y}\right)+\text { h.c. } \\
& L_{y}=-\sqrt{3} \mathrm{i} c_{x z}^{\dagger} c_{z^{2}}+\mathrm{i}\left(c_{y z}^{\dagger} c_{x y}+c_{x z}^{\dagger} c_{x^{2}-y^{2}}\right)+\text { h.c., } \\
& L_{z}=-\mathrm{i}\left(c_{y z}^{\dagger} c_{x z}+2 c_{x y}^{\dagger} c_{x^{2}-y^{2}}\right)+\text { h.c.. }
\end{aligned}
$$

In the basis $\left(d_{y z, \uparrow}, d_{y z, \downarrow}, d_{x z, \uparrow}, d_{x z, \downarrow}, d_{x y, \uparrow}, d_{x y, \downarrow}, d_{z^{2}, \uparrow}\right.$, $\left.d_{z^{2}, \downarrow}, d_{x^{2}-y^{2}, \uparrow}, d_{x^{2}-y^{2}, \downarrow}\right)$, one can construct the explicit matrix representation of the Hamiltonian $H$ :

$\left(\begin{array}{cccccccccc}\Delta_{t} & 0 & \frac{\mathrm{i} \lambda}{2} & 0 & 0 & -\frac{\lambda}{2} & 0 & -\frac{\sqrt{3} \mathrm{i} \lambda}{2} & 0 & -\frac{\mathrm{i} \lambda}{2} \\ 0 & \Delta_{t} & 0 & -\frac{\mathrm{i} \lambda}{2} & \frac{\lambda}{2} & 0 & -\frac{\sqrt{3} \mathrm{i} \lambda}{2} & 0 & -\frac{\mathrm{i} \lambda}{2} & 0 \\ -\frac{\mathrm{i} \lambda}{2} & 0 & \Delta_{t} & 0 & 0 & \frac{\mathrm{i} \lambda}{2} & 0 & \frac{\sqrt{3} \lambda}{2} & 0 & -\frac{\lambda}{2} \\ 0 & \frac{\mathrm{i} \lambda}{2} & 0 & \Delta_{t} & \frac{\mathrm{i} \lambda}{2} & 0 & -\frac{\sqrt{3} \lambda}{2} & 0 & \frac{\lambda}{2} & 0 \\ 0 & \frac{\lambda}{2} & 0 & -\frac{\mathrm{i} \lambda}{2} & \Delta_{t} & 0 & 0 & 0 & \mathrm{i} \lambda & 0 \\ -\frac{\lambda}{2} & 0 & -\frac{\mathrm{i} \lambda}{2} & 0 & 0 & \Delta_{t} & 0 & 0 & 0 & -\mathrm{i} \lambda \\ 0 & \frac{\sqrt{3} \mathrm{i} \lambda}{2} & 0 & -\frac{\sqrt{3} \lambda}{2} & 0 & 0 & 0 & 0 & 0 & 0 \\ \frac{\sqrt{3} \mathrm{i} \lambda}{2} & 0 & \frac{\sqrt{3} \lambda}{2} & 0 & 0 & 0 & 0 & 0 & 0 & 0 \\ 0 & \frac{\mathrm{i} \lambda}{2} & 0 & \frac{\lambda}{2} & -\mathrm{i} \lambda & 0 & 0 & 0 & 0 & 0 \\ \frac{\mathrm{i} \lambda}{2} & 0 & -\frac{\lambda}{2} & 0 & 0 & \mathrm{i} \lambda & 0 & 0 & 0 & 0\end{array}\right)$,

where $\Delta_{t}=\epsilon_{t_{2 g}}-\epsilon_{e_{g}}$ is a positive constant measuring the energy gap between the $e_{g}$ and the $t_{2 g}$ orbitals.
The key effect of the SOC is the entanglement between spin and orbital degrees of freedom in the electronic wavefunctions. However, in the case of a tetrahedral CF splitting favoring an $e_{g}^{1}$ configuration, the only non-vanishing matrix elements are the off-diagonal SOC terms between the $e_{g}$ and $t_{2 g}$ manifolds. Such terms contribute with an energy correction to the dominant energy scale that is proportional to the ratio $\lambda^{2} / \Delta_{t}$. The evolution of the energy levels obtained by diagonalizing the matrix of Eq. (7) is schematically shown in Fig. 1c. As one can see, the SOC affects neither the degree of degeneracy nor the hierarchy of the lower four-fold degenerate levels. Conversely, the SOC can split the $t_{2 g}$ orbitals, which are characterized by an unquenched projection of $L$. Finally, we point out that, as a consequence of the spin-orbital mixing provided by the SOC, a non-vanishing orbital angular momentum arises, which is fully isotropic.

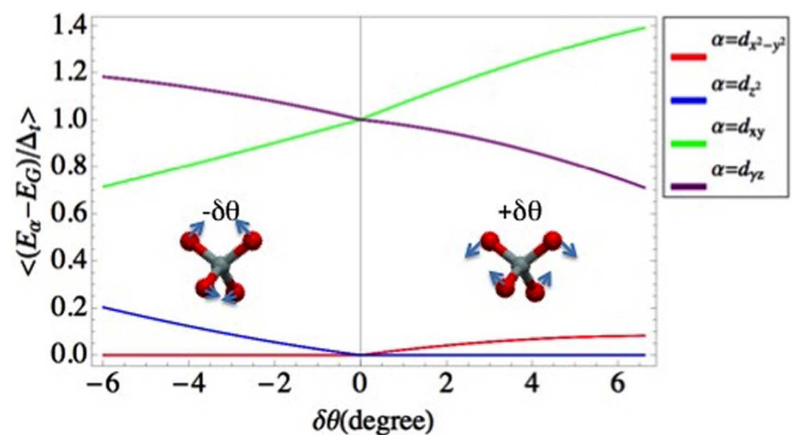

Fig. 2. (a) Evolution of the $d$ energy levels $\left(E_{\alpha}\right)$ for tetrahedra that are squeezed (left) or squashed (right) along the $z$ direction, as a function of the deviation $\delta_{\theta}$ from the ideal $\theta=109.5^{\circ}$. We denote by $E_{G}$ the energy of the lowest energy level, which is $d_{z^{2}}$ for $\delta_{\theta}>0$ and $d_{x^{2}-y^{2}}$ for $\delta_{\theta}<0$.

\section{Spin-orbit in the presence of tetrahedral distortions}

Let us consider now the interplay between the SOC and the tetrahedron distortions and how they affect a spin-orbit entangled ground state. In doing that, we are assuming that the tetrahedron is firstly deformed as to remove the orbital degeneracy and then achieves a new lower energy ground state. Then, we proceed starting from the limit of zero spin-orbit coupling to investigate how the energy levels are affected when we turn on the spin-orbit interaction.

In order to do that, we consider a local deformation related to a specific static normal mode associated to the tetrahedral symmetry. In particular, we choose the one corresponding to deformations of the triangular shapes that can be obtained by varying the O-TM-O angle, without affecting the TM-O length, which is left as a free parameter. These deformations can be visualized as a squashed $\left(\delta_{\theta}>0\right)$ or a squeezed $\left(\delta_{\theta}<0\right)$ tetrahedron along the $z$ direction and are schematically depicted in Fig. 2. The distorted tetrahedral environment induces a 
partial lifting of the orbital degeneracy even in the absence of SOC. To get an estimate of the modified on-site energies $E_{\alpha}$, we calculate the potential energy that the $d^{1}$ electron, treated as a point charge located at the origin, experiences because of four negative point charges located at the $\mathrm{O}$ sites. By evaluating the averages of the potential over the $d$ orbitals, we obtain the dependence on the deviation angle of the on-site energies, which are reported in Fig. 2 in units of $\Delta_{t}$.

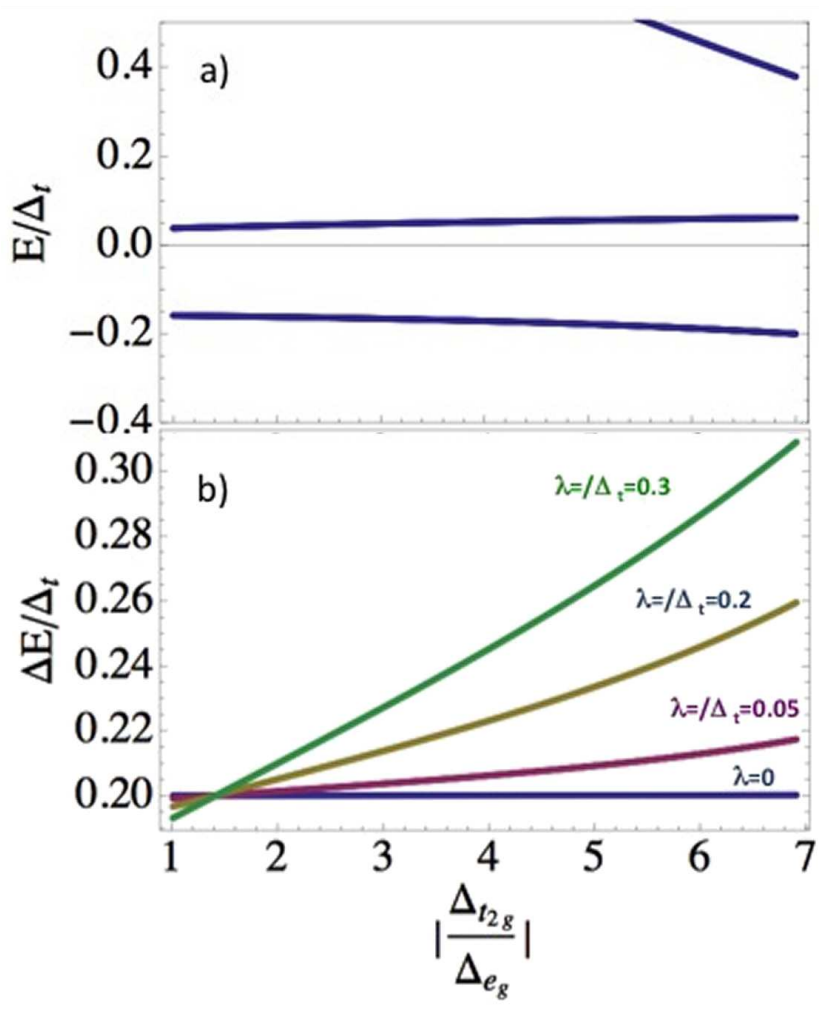

Fig. 3. (a) Evolution of the lowest energy eigenvalues of the Hamiltonian in Eq. (1) as functions of the ratio between $\Delta_{t_{2 g}}$ and $\Delta_{e_{g}}$ for a representative value of $\lambda / \Delta_{t}=0.2$. (b) Evolution of the energy difference $\Delta E$ among the two lowest eigenvalues in panel (a) for various values of $\lambda / \Delta_{t}$.

As one can see, in the case of both squashed and squeezed tetrahedra, the degeneracy in the $e_{g}$ subspace is lifted. In the first case, the $d_{z^{2}}$ orbital is shifted lower with respect to the $d_{x^{2}-y^{2}}$ one by an amount $\Delta_{e_{g}}$, while the opposite ordering occurs for a squeezed tetrahedron. In the $t_{2 g}$ sector, an energy gap $\Delta_{t_{2 g}}$ separates the $d_{x y}$ orbital from the manifold of degenerate $d_{y z}$ and $d_{x z}$ ones. We observe that the relative ratio $\Delta_{t_{2 g}} / \Delta_{e_{g}}$ is always greater than 1 in both regions and raises up to several units when the deformation angle increases. Hereafter, we use the ratio $\Delta_{t_{2 g}} / \Delta_{e_{g}}$ to parametrize the degree of distortion instead of the angular deviation $\delta \theta$.
In the following, we analyze the competition between the SOC and the tetrahedral distortions, for the particular case of squashed tetrahedra. We use a typical value of $\Delta_{e_{g}} / \Delta_{t}=0.2$ and follow the evolution of the lowest energy eigenvalues as functions of $\lambda / \Delta_{t}$ and $\Delta_{t_{2 g}} / \Delta_{e_{g}}$. The results are reported in Fig. 3. We observe that the original gap $\Delta_{e_{g}}$ is amplified by the SOC and grows both with the ratio $\Delta_{t_{2 q}} / \Delta_{e_{g}}$ and with $\lambda / \Delta_{t}$ (see Fig. $3 \mathrm{~b}$ ). We deduce that, in the presence of tetrahedral distortions, the SOC can play a relevant role in setting the energy gap among the lowest energy levels that are involved in the exchange processes. We also observe that, due to the symmetry breaking in the orbital space, the emerging angular momentum is highly anisotropic due to the preferential action of the $L_{x}$ and $L_{y}$ components over the CF-selected $d_{z^{2}}$ ground state.

\section{Conclusions}

Moderate values of the ratio $\lambda / \Delta_{t}$ have been considered as source of unquenched angular momentum in $d^{1}$ systems characterized by tetrahedral coordination. The interplay between SOC and CF parameters may enhance the gap within the set of lowest energy levels in the presence of tetrahedral distortions and leads to magnetic anisotropy. This mechanism is expected to play a fundamental role in the magnetic exchange processes of heavy transition-metal oxides in the $d^{1}$ configuration.

\section{References}

[1] M. Cuoco, F. Forte, C. Noce, Phys. Rev. B 73, 094428 (2006); M. Cuoco, F. Forte, C. Noce, Phys. Rev. B 74, 195124 (2006).

[2] M. Cuoco, F. Forte, C. Noce, Phys. Rev. B 82, 155104 (2010).

[3] G. Chen, Y. B. Kim, Phys. Rev. B 87, 165120 (2013).

[4] A. Avella, P. Horsch, A. M. Oleś, Phys. Rev. B 87, 045132 (2013).

[5] M. Malvestuto, V. Capogrosso, E. Carleschi, L. Galli, E. Gorelov, E. Pavarini, R. Fittipaldi, F. Forte, M. Cuoco, A. Vecchione, F. Parmigiani, Phys. Rev. B 88, 195143 (2013).

[6] V. Granata, L. Capogna, F. Forte, M.B. Lepetit, R. Fittipaldi, A. Stunault, M. Cuoco, A. Vecchione, Phys. Rev. B 93, 115128 (2016).

[7] G. Jackeli, G. Khaliullin, Phys. Rev. Lett. 102 , 017205 (2009).

[8] I.I. Mazin, H.O. Jeschke, K. Foyevtsova, R. Valentí, D.I. Khomskii, Phys. Rev. Lett. 109, 197201 (2012).

[9] F. Forte, M. Cuoco, J. van den Brink, Phys. Rev. B 88, $144422(2013)$.

[10] W. Witczak-Krempa, Gang Chen, Yong Baek Kim, L. Balents, Annu. Rev. Condens. Matter Phys. 5, 57 (2014). 
[11] N. Nembrini, S. Peli, F. Banfi, G. Ferrini, Y. Singh, P. Gegenwart, R. Comin, K. Foyevtsova, A. Damascelli, A. Avella, C. Giannetti, Phys. Rev. B 94, 201119(R) (2016).

[12] D. Mandrus, J.R. Thompson, R. Gaal, L. Forro, J.C. Bryan, B.C. Chakoumakos, L.M. Woods, B.C. Sales, R.S. Fishman, V. Keppens, Phys. Rev. B 63, 195104 (2001).

[13] Y.G. Shi, Y.F. Guo, S. Yu, M. Arai, A.A. Belik, A. Sato, K. Yamaura, E. Takayama-Muromachi, H. F. Tian, H.X. Yang, J.Q. Li, T. Varga, J.F. Mitchell, S. Okamoto, Phys. Rev. B 80, 161104 (2009).

[14] J. Yamaura, K. Ohgushi, H. Ohsumi, T. Hasegawa, I. Yamauchi, K. Sugimoto, S. Takeshita, A. Tokuda, M. Takata, M. Udagawa, M. Takigawa, H. Harima, T. Arima, Z. Hiroi, Phys. Rev. Lett. 108, 247205 (2012).
[15] S. Calder, V.O. Garlea, D.F. McMorrow, M.D. Lumsden, M.B. Stone, J.C. Lang, J.-W. Kim, J.A. Schlueter, Y.G. Shi, K. Yamaura, Y.S. Sun, Y. Tsujimoto, A.D. Christianson, Phys. Rev. Lett. 108, 257209 (2012).

[16] D.J. Groenendijk, C. Autieri, J. Girovsky, M. Carmen Martinez-Velarte, N. Manca, G. Mattoni, A.M.R.V.L. Monteiro, N. Gauquelin, J. Verbeeck, A.F. Otte, M. Gabay, S. Picozzi, A.D. Caviglia, Phys. Rev. Lett. 119, 256403 (2017).

[17] Y.J. Song, Kyo-Hoon Ahn, Kwan-Woo Lee, W.E. Pickett, Phys. Rev. B 90, 245117 (2014).

[18] F. Forte, D. Guerra, C. Autieri, A. Romano, C. Noce, Phys. B: Cond. Matter 537, 184 (2018). 